\title{
A FEW REMARKS ON QUADRATIC HARNESSES
}

\author{
PAWEŁ J. SZABŁOWSKI
}

\begin{abstract}
We analyze and partially solve system of recurrences that can be derived from the properties of martingale orthogonal polynomials that characterize quadratic harnesses $(\mathrm{QH})$. We also specify conditions for the existence of moments of one dimensional distribution for large classes of quadratic harnesses that are also Markov processes complementing earlier results.
\end{abstract}

\section{INTRODUCTION}

In the series of papers [2], [4, [5], [3, [6], 7], 8] Bryc and Wesołowski supported from time to time by Matysiak have defined and analyzed a wide class of stochastic processes that they have called Quadratic Harnesses (briefly QH). While performing this analysis they have obtained a system of 5 recurrence equations depending on 5 parameters.

In this paper we derive some general properties of this system of equations as well as partially solve it. More precisely we are able to find solutions of these recurrences mostly when 2 out of 5 parameters are set to zero.

In order to make paper complete we present the origins and interpretation of these recurrences. We have placed them in the appendix. We also pose some open problems that may encourage more gifted researches to continue our investigations.

The paper is organized as follows.

In the next Section 2 we study these equations partially solving them or at least providing conditions for the existence of one dimensional measure that is identifiable by moments. On the way we use results of the special auxiliary Section 4 that we have placed at the end of the paper. Section 2 contains our main results concerning integrability and existence of sequence of OMP for a given $\mathrm{QH}$ characterized by 5 parameters.

Next short Section 3 contains some remarks concerning the results and presents some open problem that appeared while writing the paper. Final, fifth Section 5 contains lengthy proofs.

At the end we present an appendix $\mathrm{A}$. We give there a definition and basic properties of quadratic harnesses. That is we recollect results of works of Bryc and others. We sketch an alternative derivation of the system of 5 iterative equations that must be satisfied if sequence of OMP is to exist for a given $\mathrm{QH}$. This derivation is believed to be simpler than the original one given in 5 .

Date: June 2012

2000 Mathematics Subject Classification. Primary 39A10, 39A13; Secondary 33D45, 60G44.

Key words and phrases. system of difference equations, orthogonal polynomials, $q$-series, 3 term recurrence.

The author would like to thank unknown referee for pointing out numerous mistakes, inaccuracies and misprints of the first version of the paper. 
Throughout the paper we use traditional notation used in $q$-series theory. $q$ is a parameter and we assume $q \in(-1,1]$. In particular we denote:

$$
[0]_{q}=0 ;[n]_{q}=1+q+\ldots+q^{n-1},[n]_{q} !=\prod_{j=1}^{n}[j]_{q},
$$

with $[0]_{q} !=1$, and

$$
\left[\begin{array}{l}
n \\
k
\end{array}\right]_{q}=\left\{\begin{array}{cl}
\frac{[n]_{q} !}{[n-k]_{q} ![k]_{q} !} & , \quad n \geq k \geq 0, \\
0 & , \quad \text { otherwise. }
\end{array}\right.
$$

The system of recurrences that we are going to study is the following:

$$
\begin{aligned}
\tau \alpha_{n} \alpha_{n+1}+q \alpha_{n} \beta_{n+1}+\sigma \beta_{n} \beta_{n+1} & =\alpha_{n+1} \beta_{n}, \\
\tau \varepsilon_{n-1} \varepsilon_{n}+q \varepsilon_{n} \varphi_{n-1}+\sigma \varphi_{n} \varphi_{n-1} & =\varepsilon_{n-1} \varphi_{n},
\end{aligned}
$$

$\theta \alpha_{n}+\eta \beta_{n}+\tau \alpha_{n}\left(\gamma_{n}+\gamma_{n+1}\right)+\sigma \beta_{n}\left(\delta_{n}+\delta_{n+1}\right)+q\left(\alpha_{n} \delta_{n+1}+\beta_{n} \gamma_{n}\right)=\beta_{n} \gamma_{n+1}+\alpha_{n} \delta_{n}$,

$\theta \varepsilon_{n}+\eta \varphi_{n}+\tau \varepsilon_{n}\left(\gamma_{n}+\gamma_{n-1}\right)+\sigma \varphi_{n}\left(\delta_{n-1}+\delta_{n}\right)+q\left(\varphi_{n} \gamma_{n}+\delta_{n-1} \varepsilon_{n}\right)=\varepsilon_{n} \delta_{n}+\varphi_{n} \gamma_{n-1}$,

(1.5) $1+\theta \gamma_{n}+\eta \delta_{n}+\tau \gamma_{n}^{2}+\sigma \delta_{n}^{2}+\tau\left(\alpha_{n-1} \varepsilon_{n}+\alpha_{n} \varepsilon_{n+1}\right)+\sigma\left(\varphi_{n} \beta_{n-1}+\beta_{n} \varphi_{n+1}\right)$

$$
+q\left(\gamma_{n} \delta_{n}+\beta_{n-1} \varepsilon_{n}+\alpha_{n} \varphi_{n+1}\right)=\gamma_{n} \delta_{n}+\beta_{n} \varepsilon_{n+1}+\varphi_{n} \alpha_{n-1} .
$$

for $n \geq 1$ with initial conditions $\alpha_{0}=\gamma_{0}=\delta_{0}=\varphi_{1}=0$ and $\beta_{0}=\varepsilon_{1}=1$.

The general task is to examine existence of solutions of this system of recurrences for all values of parameters, since these recurrences may be interesting on its own. However from the point of view of the theory of quadratic harness the most important are those ranges of parameters that lead to sets of orthogonal polynomials more specifically mostly to the polynomials from the so called Askey-Wilson scheme of polynomials.

The meaning of real sequences $\left\{\alpha_{n}\right\},\left\{\beta_{n}\right\},\left\{\gamma_{n}\right\},\left\{\delta_{n}\right\},\left\{\varepsilon_{n}\right\},\left\{\varphi_{n}\right\}$ is the following. Using these sequences we define 3 sequences of linear functions $a_{n}(t)$ $=\alpha_{n} t+\beta_{n}, b_{n}(t)=\gamma_{n} t+\delta_{n}, c_{n}(t)=\varepsilon_{n} t+\varphi_{n}$, for $t \geq 0$ so that these function sequences define a system of orthogonal polynomials defined by the following 3 -term recurrence:

$$
x p_{n}(x ; t)=a_{n}(t) p_{n+1}(x ; t)+b_{n}(t) p_{n}(x ; t)+c_{n}(t) p_{n-1}(x ; t),
$$

for all $t \geq 0, n \geq 0$. It is convenient to interpret parameter $t$ as time. Functions $a_{n}, b_{n}$ and $c_{n}$ are real and must depend on $t$ and on five parameters $\sigma, \tau, \eta, \theta$ and $q$ only.

Now let us recall from the general theory of orthogonal polynomials that the 3 -term recurrence (1.7) defines the system of polynomials orthogonal with respect to a nonnegative measure with the support consisting of infinitely many points iff for all $n \geq 1$ and $t \geq 0$ :

$$
a_{n-1}(t) c_{n}(t)>0 .
$$

For more details on the theory of orthogonal polynomials we refer the reader for example to [1] or to [11. Hence from this point of view the most interesting range of parameters is the one that guaranties fulfilment of this requirement. Further task is to solve recurrences and then having solutions find polynomials $\left\{p_{n}\right\}$ solving 
above mentioned 3-term recurrence and finally find positive measures that make these polynomials orthogonal.

In the next section we will define the some ranges of parameters $q, \tau, \sigma, \theta, \eta$ that assure that condition (1.8) is satisfied.

\section{ANALYSIS AND INTEGRABILITY}

We have the following two observations concerning the first and the second pair of the system of equations (1.1)-(1.6). The first one concerns equations (1.1) and (1.2). Both these observations are the base of the following simple remark. Notice that if the OMP sequence is to exist coefficients $a_{n}(t) \neq 0$ for all $n$ and $t$. That is sequences $\left\{\alpha_{n}\right\}$ and $\left\{\beta_{n}\right\}$ cannot vanish simultaneously. Similarly $c_{n}(t)$ cannot vanish for all $n$ and $t$.

In the sequel the crucial rôle will be played by the following sequence $\left\{\lambda_{n}\right\}_{n \geq 0}$ defined by the following recursion:

$$
\lambda_{n+1}=\frac{1+q \lambda_{n}}{1-\sigma \tau \lambda_{n}}
$$

with $\lambda_{0}=0$.

Below we present two general properties of our system of recurrences that are true provided parameters are chosen in such a way that sequence of reals $\left\{\lambda_{n}\right\}$ defined by (2.1) and sequence of matrices defined by (2.2) do exist. These properties will lead to certain equivalent reformulation of recurrences simplifying imposition of the condition (1.8). Then we will formulate appropriate ranges for parameters $q, \tau, \sigma$, $\theta, \eta$.

Hence for the moment let us assume that parameters $\sigma \tau$ and $q$ are chosen in such away that $\forall n \geq 1 \lambda_{n} \neq 0$. Lemma 1, below provides necessary conditions for this to happen.

Theorem 1. $\forall n \geq 0: \alpha_{n}=\sigma \lambda_{n} \beta_{n}$ and $\varphi_{n}=\tau \lambda_{n-1} \varepsilon_{n}$.

Proof. Firstly assume that $\sigma=0$ and let us consider equation (1.1). Put $n=0$. Then we see that $\alpha_{1}=0$. But this means that $\beta_{1} \neq 0$. Now let us put $n=1$ in (1.1). We have $0=\alpha_{2} \beta_{1}$. Hence we deduce that $\alpha_{2}=0$ and $\beta_{2} \neq 0$. And so on by induction we deduce that in this case $\alpha_{n}=0$ and $\beta_{n} \neq 0$. In the similar way we show that if $\tau=0$ then $\varphi_{n}=0$ and $\varepsilon_{n} \neq 0$. Now assume that $\sigma \neq 0$ and consider equation (1.1). Notice that for $n=0$ this equation yields $\alpha_{1}=\sigma \beta_{1}$. Since $\alpha_{1}^{2}+\beta_{1}^{2} \neq 0$ we see that $\beta_{1} \neq 0$. We will now deduce by induction. Assume that $\alpha_{n}=\sigma \lambda_{n} \beta_{n}$ and $\beta_{n} \neq 0$. Considering equation (1.1) we have

$$
\alpha_{n+1}\left(1-\sigma \tau \lambda_{n}\right) \beta_{n}=\left(1+q \lambda_{n}\right) \sigma \beta_{n} \beta_{n+1} .
$$

Since $\beta_{n} \neq 0$ we deduce that $\alpha_{n+1}=\sigma \lambda_{n+1} \beta_{n+1}$ and $\beta_{n+1} \neq 0$ if $\lambda_{n+1} \neq 0$. We reason similarly in the case of equation (1.2) and sequences $\varphi_{n}$ and $\varepsilon_{n}$.

Further let us consider equations (1.3) and (1.4).

Again for the moment let us assume that the parameters $\sigma, \tau, q$ are chosen in such a way that matrices defined by (2.2) are non-singular. Lemma 1, below will present necessary conditions for this to happen. We have the following result. 
Theorem 2. Let us define the following sequence of $2 \times 2$ matrices for $n \geq 1$ :

$$
\begin{aligned}
A_{n} & =\left[\begin{array}{cc}
1-\tau \sigma \lambda_{n} & -\sigma\left(1+q \lambda_{n}\right) \\
-\tau\left(1+q \lambda_{n}\right) & 1-\sigma \tau \lambda_{n}
\end{array}\right], \\
B_{n} & =\left[\begin{array}{cc}
q+\sigma \tau \lambda_{n} & \sigma\left(1-\lambda_{n}\right) \\
\tau\left(1-\lambda_{n}\right) & q+\sigma \tau \lambda_{n}
\end{array}\right], \\
C_{n} & =\left[\begin{array}{cc}
\sigma \lambda_{n} & 1 \\
1 & \tau \lambda_{n}
\end{array}\right] .
\end{aligned}
$$

Then sequences $\left\{\gamma_{n}\right\}$ and $\left\{\delta_{n}\right\}$ are given by the following formulae for $n \geq 0$ :

$$
\left[\begin{array}{l}
\gamma_{n+1} \\
\delta_{n+1}
\end{array}\right]=\sum_{k=0}^{n}\left(\prod_{j=k+1}^{n} \Xi_{j}\right) w_{k}
$$

where we denoted $w_{k}=A_{k}^{-1} C_{k}\left[\begin{array}{l}\theta \\ \eta\end{array}\right], \Xi_{k}=A_{k}^{-1} B_{k}$ for $k>0$. In 2.5 we set $\prod_{k=n+1}^{n} \Xi_{k}=I$ and $\left[\begin{array}{l}\gamma_{1} \\ \delta_{1}\end{array}\right]=w_{0}$.

Let us denote $\chi_{n}=\beta_{n-1} \varepsilon_{n}$. Then sequence $\chi_{n}$ satisfies the following recursion:

$\left(q+\sigma \tau-\sigma \tau\left(1-\lambda_{n-1}\right)^{2}\right) \chi_{n}+1+\theta \gamma_{n}+\tau \gamma_{n}^{2}+\eta \delta_{n}+\sigma \delta_{n}^{2}-(1-q) \gamma_{n} \delta_{n}=\left(1-\sigma \tau\left(2 \lambda_{n}+q \lambda_{n}^{2}\right)\right) \chi_{n+1}$, with $\chi_{1}=1$.

Proof. Dividing both sides of (1.3) by $\beta_{n}$ and keeping in mind that $\frac{\alpha_{n}}{\beta_{n}}=\sigma \lambda_{n}$ we get:

$$
\gamma_{n+1}\left(1-\tau \sigma \lambda_{n}\right)-\sigma\left(1+q \lambda_{n}\right) \delta_{n+1}=\left(\tau \sigma \lambda_{n}+q\right) \gamma_{n}+\sigma\left(1-\lambda_{n}\right) \delta_{n}+\theta \sigma \lambda_{n}+\eta .
$$

Dividing both sides of (1.4) by $\varepsilon_{n}$ and keeping in mind that $\frac{\varphi_{n}}{\varepsilon_{n}}=\tau \lambda_{n-1}$ we get after shifting indices by 1 :

$$
\delta_{n+1}\left(1-\sigma \tau \lambda_{n}\right)-\tau\left(1+q \lambda_{n}\right) \gamma_{n+1}=\tau\left(1+q \lambda_{n}\right) \gamma_{n}+\left(\sigma \tau \lambda_{n}-1\right) \delta_{n}+\theta+\tau \lambda_{n} \eta .
$$

As the result we obtain the following new vector form of equations (1.3) and (1.4):

$$
\left[\begin{array}{c}
\gamma_{n+1} \\
\delta_{n+1}
\end{array}\right]=A_{n}^{-1} B_{n}\left[\begin{array}{l}
\gamma_{n} \\
\delta_{n}
\end{array}\right]+A_{n}^{-1} C_{n}\left[\begin{array}{l}
\theta \\
\eta
\end{array}\right]
$$

with $\left[\begin{array}{l}\gamma_{0} \\ \delta_{0}\end{array}\right]=\left[\begin{array}{l}0 \\ 0\end{array}\right]$ for $n \geq 0$. Proof of (2.5) is by induction. We have on the left hand side: $\left[\begin{array}{c}\gamma_{n+1} \\ \delta_{n+1}\end{array}\right]=\sum_{k=0}^{n}\left(\prod_{j=k+1}^{n} \Xi_{j}\right) w_{k}$, while right hand side is $\Xi_{n} \sum_{k=0}^{n-1}\left(\prod_{j=k+1}^{n-1} \Xi_{j}\right) w_{k}+w_{n}=\sum_{k=0}^{n-1}\left(\prod_{j=k+1}^{n} \Xi_{j}\right) w_{k}+w_{n}=\sum_{k=0}^{n}\left(\prod_{j=k+1}^{n} \Xi_{j}\right) w_{k}$. Finally let us consider equation given by (1.5) and (1.6). Taking $\alpha_{n}=\sigma \lambda_{n} \beta_{n}$ and $\varphi_{n+1}=\tau \lambda_{n} \varepsilon_{n+1}$ and denoting $\chi_{n}=\beta_{n-1} \varepsilon_{n}$ we get (2.6) with $\chi_{1}=1$.

As a corollary we have the following observation.

\section{Corollary 1.}

$$
1+\theta \gamma_{n}+\tau \gamma_{n}^{2}+\eta \delta_{n}+\sigma \delta_{n}^{2}-(1-q) \gamma_{n} \delta_{n}=\boldsymbol{\mu}^{T}\left(\left(\mathbf{D}_{n}+\mathbf{D}_{n}^{T}\right) / 2+\mathbf{D}_{n}^{T} \boldsymbol{\Delta} \mathbf{D}_{n}\right) \boldsymbol{\mu},
$$

where we denoted $\boldsymbol{\mu}=(\theta, \eta)^{T}, \mathbf{D}_{n}=\sum_{k=0}^{n}\left(\prod_{j=k+1}^{n} \Xi_{j}\right) A_{k}^{-1} C_{k}$ and

$$
\Delta=\left[\begin{array}{cc}
\tau & -(1-q) / 2 \\
-(1-q) / 2 & \sigma
\end{array}\right] \text {. }
$$


Proof. Notice that from (2.5) it follows that vector $\left(\gamma_{n}, \delta_{n}\right)^{T}$ is a linear form of parameters $\theta$ and $\eta$ more precisely that $\left(\gamma_{n}, \delta_{n}\right)^{T}=\mathbf{D}_{n} \boldsymbol{\mu}$. The final form follows the fact that $\boldsymbol{\mu}^{T} \mathbf{A} \boldsymbol{\mu}=\boldsymbol{\mu}^{T}\left(\mathbf{A}+\mathbf{A}^{T}\right) \boldsymbol{\mu} / 2$ for any matrix $\mathbf{A}$.

Let us now redefine orthogonal polynomials $p_{n}$ originally defined by (1.7). Namely let us consider new polynomials $M_{n}(y \mid t, \sigma, \tau, \theta, \eta, q)$ briefly denoted $M_{n}(y)$ related to polynomials $p_{n}$ in the following way:

$$
M_{n}(y)=\frac{\prod_{j=0}^{n-1} a_{j}(t)}{t^{n / 2}} p_{n}(y \sqrt{t} ; t) .
$$

Polynomials $M_{n}(y)$ although have less straightforward probabilistic interpretation are easier to analyze. We have the following simple observation.

Proposition 1. Polynomials $\left\{M_{n}\right\}$ satisfy the following 3-term recurrence:

$y M_{n}(y)=M_{n+1}(y)+\left(\gamma_{n} \sqrt{t}+\delta_{n} / \sqrt{t}\right) M_{n}(y)+\chi_{n}\left(1+\sigma \lambda_{n-1} t+\tau \lambda_{n-1} / t+\sigma \tau \lambda_{n-1}^{2}\right) M_{n-1}(y)$,

with $M_{-1}(y)=0, M_{0}(y)=1$.

Proof. Multiplying both sides of (1.7) by $\prod_{j=0}^{n-1} a_{j}(t) / t^{(n+1) / 2}$ we get

$$
\begin{gathered}
\prod_{j=0}^{n-1} a_{j}(t) \frac{x}{\sqrt{t}} \frac{p_{n}(x ; t)}{t^{n / 2}}=\prod_{j=0}^{n} a_{j}(t) \frac{p_{n+1}(x ; t)}{t^{(n+1) / 2}}+\prod_{j=0}^{n-1} a_{j}(t) \frac{1}{t^{(n+1) / 2}} b_{n}(t) p_{n}(x, t) \\
+a_{n-1}(t) \frac{c_{n}(t)}{t} \prod_{j=0}^{n-2} a_{j}(t) \frac{p_{n-1}(x ; t)}{t^{(n-1) / 2}}
\end{gathered}
$$

Now it remains to change variable $x \longrightarrow y \sqrt{t}$, use Theorem 1 and multiply $\left(\sigma \lambda_{n-1} t+\right.$ 1) $\left(1+\tau \lambda_{n-1} / t\right)$.

Now let us recall Favard's Theorem that assures that the infinitely supported measure that makes polynomials $M_{n}$ orthogonal is positive iff $\forall t>0, n \geq 0$ :

$$
\chi_{n}\left(1+\sigma \lambda_{n-1} t+\tau \lambda_{n-1} / t+\sigma \tau \lambda_{n-1}^{2}\right)>0 .
$$

First observation is that both $\forall n \geq 0: \sigma \chi_{n} \lambda_{n-1} \geq 0$ and $\tau \chi_{n} \lambda_{n-1} \geq 0$ from which it immediately follows that $\sigma \tau \geq 0$. However for the function $\left(1+\sigma \lambda_{n-1} t+\tau \lambda_{n-1} / t+\right.$ $\left.\sigma \tau \lambda_{n-1}^{2}\right)$ not to change sign for $t>0$ one needs that both of its roots be nonpositive which implies that both $\sigma \lambda_{n-1}$ and $\tau \lambda_{n-1}$ be non-negative. Notice further also that following properties of the sequence $\left\{\lambda_{n}\right\}$ we deduce that the sequence $\left\{\left(1+\sigma \lambda_{n-1} t+\tau \lambda_{n-1} / t+\sigma \tau \lambda_{n-1}^{2}\right)\right\}$ is bounded and positive for every $t>0$. Hence if this measure has to be nonnegative the sequence $\left\{\chi_{n}\right\}$ has to be additionally positive. Taking into account the fact that from assertion iv) of Proposition 3 it follows that if $q>1-2 \sqrt{\sigma \tau}$ then the sequence $\lambda_{n}$ changes sign infinitely often we deduce that then the sequence $\left\{\chi_{n}\left(1+\sigma \lambda_{n-1} t+\tau \lambda_{n-1} / t+\sigma \tau \lambda_{n-1} \lambda_{n}\right)\right\}$ cannot be non-negative for all $t>0$. Hence we will consider only the case $q \leq 1-2 \sqrt{\sigma \tau}$.

Remark 1. Notice that redefining variables $x \longrightarrow y \sqrt{t}$ allows to see additional symmetries of the distribution that makes polynomials $p_{n}$ orthogonal. Namely from formula (2.9) it follows that if $\sigma=\tau$ and $\eta=\theta$ then the we have $\gamma_{n}=\delta_{n}$ for all $n \geq 0$ and consequently that the change of time $t \longrightarrow 1 / t$ does not change polynomials $M_{n}(y, t)$. 
To clear the situation concerning existence of the sequence $\left\{\lambda_{n}\right\}_{n>0}$, and invertibility of matrices $\left\{A_{n}\right\}_{n>0}$ we have the following lemma being a direct consequence of Proposition 3 presented below in Section 4 .

Lemma 1. i) If $1 \geq \sigma \tau \geq 0$ and $-1<q \leq 1-2 \sqrt{\sigma \tau}$ then $\forall n \geq 0: \lambda_{n} \geq 0$ and

$$
\lambda_{n} \longrightarrow y(q, \sigma \tau) \leq \frac{2}{1-q} \leq \frac{1}{\sqrt{\sigma t}}
$$

as $n \longrightarrow \infty$, where $y(q, \sigma \tau)$ is given by 4.1$)$. Moreover if $-1<q<1-2 \sqrt{\sigma \tau}$ then this convergence is exponential i.e. $\left|\lambda_{n}-y(q, \sigma \tau)\right|<C^{n}(q, \sigma \tau)$ where $C \in[0,1)$.

ii) Matrices $A_{n}$ defined by (2.2) are non-singular.

iii) $\forall n \geq 1$ :

$$
\left(1-\sigma \tau\left(2 \lambda_{n}+q \lambda_{n}^{2}\right)\right)>0 .
$$

If $q+\sigma \tau \geq 0$ then

$$
\left(q+\sigma \tau-\sigma \tau\left(1-\lambda_{n-1}\right)^{2}\right) \geq 0,
$$

for all $n \geq 1$ and if $q+\sigma \tau<0$ then

$$
\left(q+\sigma \tau-\sigma \tau\left(1-\lambda_{n-1}\right)^{2}\right)<0,
$$

for all $n \geq 1$.

iv) If $-1<q \leq 1-2 \sqrt{\sigma \tau}$ then $\frac{\left(q+\sigma \tau-\sigma \tau\left(1-\lambda_{n-1}\right)^{2}\right)}{\left(1-\sigma \tau\left(2 \lambda_{n}+q \lambda_{n}^{2}\right)\right)} \longrightarrow D(q, \sigma \tau)$, as $n \longrightarrow \infty$, where

$$
D(q, \sigma \tau)=\frac{4(q+\sigma \tau)}{\left(1+q+\sqrt{(1-q)^{2}-4 \sigma \tau}\right)^{2}} .
$$

For $-1<q<1-2 \sqrt{\sigma \tau}$ we have $|D(q, \sigma \tau)|<1$.

v) $-1<q<1-2 \sqrt{\sigma \tau}$ and $\theta=\eta=0$ then sequence $\chi_{n} \longrightarrow \frac{\left(1-q+\sqrt{(1-q)^{2}-4 \sigma \tau}\right)}{2 \sqrt{(1-q)^{2}-4 \sigma \tau}}$ as $n \longrightarrow \infty$. Moreover if $q+\sigma \tau \geq 0$ then $\forall n \geq 1: \chi_{n} \geq 0$.

Proof. Proved is moved to Section 5 .

Let us recall also Theorem 2.5.5 of [9] assuring that the measure that makes polynomials $\left\{M_{n}\right\}$ orthogonal is unique and compactly supported if for every $t>0$ sequences $\left\{\left(\gamma_{n} t+\delta_{n}\right)\right\}$ and $\left\{\chi_{n}\left(1+\sigma \lambda_{n-1} t+\tau \lambda_{n-1} / t+\sigma \tau \lambda_{n-1}^{2}\right)\right\}$ are bounded. Hence the mentioned above theorem requires in fact only that sequences $\left\{\gamma_{n}, \delta_{n}, \chi_{n}\right\}$ are bounded and sequence $\left\{\chi_{n}\right\}$ be positive (to make the measure positive).

Proposition below lists several easy cases when almost full solution is possible. The other more complicated cases require separate analysis and treatment.

Proposition 2. i) If $\tau=\theta=0$, then $\lambda_{n}=[n]_{q}, \gamma_{n}=[n]_{q} \eta, \delta_{n}=0, \quad, \chi_{n}=[n]_{q}$ for $n \geq 0$ or if $\sigma=\eta=0$, then $\lambda_{n}=[n]_{q}, \gamma_{n}=0, \quad \delta_{n}=[n]_{q} \theta, \chi_{n}=[n]_{q}$ for $n \geq 0$.

ii) If $\tau=\eta=0$ then $\lambda_{n}=[n]_{q}, \gamma_{n}=[n]_{q}\left([n]_{q}+[n-1]_{q}\right) \theta \sigma, \delta_{n}=[n]_{q} \theta, \chi_{n}=$ $[n]_{q}+[n-1]_{q}^{2}[n]_{q} \theta^{2} \sigma$ or if $\theta=\sigma=0$, then $\gamma_{n}=[n]_{q} \eta, \delta_{n}=[n]_{q}\left([n-1]_{q}+[n]_{q}\right) \eta \tau$, $\chi_{n}=[n]_{q}+[n-1]_{q}^{2}[n]_{q} \eta^{2} \tau$, for $n \geq 0$.

iii) If $\sigma=\tau=0$, then: $\lambda_{n}=[n]_{q}, \gamma_{n}=[n]_{q} \eta, \delta_{n}=[n]_{q} \theta$ and $\chi_{n}=[n]_{q}+[n-$ $1]_{q}[n-2] \theta \eta$, for $n \geq 0$.

iv) If $q=\sigma=0$ then $\lambda_{n}=1, \gamma_{n}=\eta, \delta_{n}=\theta+2 \eta \tau, \chi_{n}=1+\eta \theta+\eta^{2} \tau$ for $n>1$, or if $q=\tau=0$ then $\lambda_{n}=1, \gamma_{n}=\eta+2 \sigma \theta, \delta_{n}=\theta, \chi_{n}=1+\eta \theta+\theta^{2} \sigma$, for $n \geq 0$. 
v) If $q=-\sigma \tau$, then $\lambda_{n}=1, \gamma_{n}=\frac{\eta+2 \theta \sigma+\eta \sigma \tau}{(1-\sigma \tau)^{2}}, \delta_{n}=\frac{\theta+2 \eta \tau+\theta \sigma \tau}{(1-\sigma \tau)^{2}}$ and $\chi_{1}=\frac{1}{(1-\sigma \tau)^{2}}$ and $\chi_{n}=\frac{1}{(1-\sigma \tau)^{2}}+\frac{(\eta+\theta \sigma)(\theta+\eta \tau)}{(1-\sigma \tau)^{4}}$, for $n \geq 0$.

vi) If $q=1-2 \sqrt{\sigma \tau}$, then $\lambda_{n}=\frac{n}{1+(n-1) \sqrt{\sigma \tau}}$. Assuming that $\theta=\eta=0$ sequence $\left\{\chi_{n}\right\}$ is given by the formula:

$$
\chi_{n}=\frac{n(1+(n-2) \sqrt{\sigma \tau})^{2}(1+(n-3) \sqrt{\sigma \tau})}{(1-\sqrt{\sigma \tau})^{2}(1+2(n-1) \sqrt{\sigma \tau})(1+2(n-2) \sqrt{\sigma \tau})},
$$

for $n \geq 1$.

Proof. Lengthy proof is shifted to Section 5 .

From the above considerations follows the following Lemma that contains observations concerning polynomials $M_{n}$ and the distribution that makes these polynomials orthogonal.

Lemma 2. i) If $-1<q<1-2 \sqrt{\sigma \tau}$ and either $q+\sigma \tau \geq 0$ and $\forall n \geq 1$ : $1+\theta \gamma_{n}+\tau \gamma_{n}^{2}+\eta \delta_{n}+\sigma \delta_{n}^{2}-(1-q) \gamma_{n} \delta_{n} \geq 0$ or $q+\sigma \tau<0$ and $\forall n>0: \chi_{n} \geq 0$ then distribution of $X_{t}$ is compactly supported for $t>0$.

ii) If $\theta=\eta=0$ and $-\sigma \tau \leq q \leq 1-2 \sqrt{\sigma \tau}$, then for every $t>0$ the distribution of $X_{t}$ is symmetric and positive. Moreover if additionally $q<1-2 \sqrt{\sigma \tau}$, then it is also compactly supported.

iii) If $\tau=\theta=0$, then the polynomials $M_{n}$ defined by (2.8) satisfy the following 3-term recurrence:

$$
y M_{n}(y)=M_{n+1}(y)+\sqrt{t} \eta[n]_{q} M_{n}(y)+[n]_{q}\left(1+\sigma t[n]_{q}\right) M_{n-1}(y),
$$

with $M_{-1}(y)=0, M_{0}(y)=1$ and if $\sigma=0$ and $\eta=0$, then the polynomials $M_{n}$ defined by (2.8) satisfy the following 3-term recurrence:

$$
y M_{n}(y)=M_{n+1}(y)+\theta[n]_{q} M_{n}(y) / \sqrt{t}+[n]_{q}\left(1+\tau[n]_{q} / t\right) M_{n-1}(y),
$$

with $M_{-1}(y)=0, M_{0}(y)=1$.

For $|q|<1$ the measures that make these polynomials orthogonal is compactly supported.

iv) If $\eta=\tau=0$, then the polynomials $M_{n}$ defined by (2.8) satisfy the following 3-term recurrence:

$$
\begin{aligned}
y M_{n}(y)= & M_{n+1}(y)+[n]_{q} \theta\left(t\left([n]_{q}+[n-1]_{q}\right) \sigma+1 / \sqrt{t}\right) M_{n}(y) \\
& +[n]_{q}\left(1+\theta^{2} \sigma[n-1]_{q}^{2}\right)\left(1+[n]_{q} \sigma t\right)\left(M_{n-1}(y),\right.
\end{aligned}
$$

with $M_{-1}(y)=0, M_{0}(y)=1$ while if $\sigma=\theta=0$ then the polynomials $M_{n}$ defined by (2.8) satisfy the following 3-term recurrence:

$$
\begin{aligned}
y M_{n}(y)= & M_{n+1}(y)+[n]_{q} \eta\left(\sqrt{t}+\left([n]_{q}+[n-1]_{q}\right) \tau / \sqrt{t}\right) M_{n}(y) \\
& +[n]_{q}\left(1+\eta^{2} \tau[n-1]_{q}^{2}\right)\left(1+[n]_{q} \tau / t\right)\left(M_{n-1}(y)\right.
\end{aligned}
$$

For $|q|<1$ the measures that make these polynomials orthogonal are compactly supported. If $q=1$ and $\sigma>0$ in case of (2.11) or

$\tau>0$ in case (2.12) measures that make polynomials orthogonal might not be indentifiable by moments. It requires special investigation.

v) If $\sigma=\tau=0$, then the polynomials $M_{n}$ defined by (2.8) satisfy the following 3-term recurrence:

$y M_{n}(y)=M_{n+1}(y)+[n]_{q}(\theta / \sqrt{t}+\eta \sqrt{t}) M_{n}(y)+\left([n]_{q}+[n-1]_{q}[n-2]_{q} \theta \eta\right) M_{n-1}(y)$, 
Again the measure that makes these polynomials orthogonal is positive if $1 \geq-\theta \eta$ and is compactly supported for $|q|<1$.

vi) If $q=\sigma=0$ then the polynomials $M_{n}$ defined by (2.8) satisfy the following 3-term recurrence:

$y M_{n}(y)=M_{n+1}(y)+(\eta \sqrt{t}+(\theta+2 \eta \tau) / \sqrt{t}) M_{n}(y)+\left(1+\eta \theta+\eta^{2} \tau\right)(1+\tau / t) M_{n-1}(y)$, with $M_{-1}(y)=0, M_{0}(y)=1$ while if $q=\tau=0$ then the polynomials $M_{n}$ defined by (2.8) satisfy the following 3-term recurrence:

$y M_{n}(y)=M_{n+1}(y)+((\eta+2 \sigma \theta) \sqrt{t}+\theta / \sqrt{t}) M_{n}(y)+\left(1+\eta \theta+\theta^{2} \sigma\right)(1+\sigma t) M_{n-1}(y)$, Again these measures is positive if either $\left(1+\eta \theta+\eta^{2} \tau\right)>0$ in the first case and $\left(1+\eta \theta+\theta^{2} \sigma\right)>0$ in the second.

vii) If $q=-\sigma \tau$, then the polynomials $M_{n}$ defined by (2.8) satisfy the following 3-term recurrence:

$$
\begin{aligned}
y M_{n}(y)= & M_{n+1}(y)+\frac{1}{(1-\sigma \tau)^{2}}((\eta+2 \theta \sigma+\eta \sigma \tau) \sqrt{t}+(\theta+2 \eta \tau+\theta \sigma \tau) / \sqrt{t}) M_{n}(y) \\
& +\frac{1}{(1-\sigma \tau)^{2}}\left(1+\frac{(\eta+\theta \sigma)(\theta+\eta \tau)}{(1-\sigma \tau)^{2}}\right)(1+\sigma \tau+\sigma t+\tau / t) M_{n-1}(y),
\end{aligned}
$$

for $n>1$ with $M_{0}(y)=1$ and

$$
M_{1}(y)=y-\frac{1}{(1-\sigma \tau)^{2}}((\eta+2 \theta \sigma+\eta \sigma \tau) \sqrt{t}+(\theta+2 \eta \tau+\theta \sigma \tau) / \sqrt{t}) .
$$

Measure that makes polynomials $M_{n}$ orthogonal is positive and compactly supported provided $\frac{(\eta+\theta \sigma)(\theta+\eta \tau)}{(1-\sigma \tau)^{2}}>-1$.

Proof. ii)-vii) follow directly from Proposition 2 and conditions for the measure orthogonalizing polynomials $M_{n}$ to be positive, while i) follows Lemma 1,ii) iv) \& v). One has to keep in mind that in order to assure that $\chi_{n} \geq 0$ and $\frac{\left(q+\sigma \tau-\sigma \tau\left(1-\lambda_{n-1}\right)^{2}\right)}{\left(1-\sigma \tau\left(2 \lambda_{n}+q \lambda_{n}^{2}\right)\right)}$ we do have to have $1+\theta \gamma_{n}+\tau \gamma_{n}^{2}+\eta \delta_{n}+\sigma \delta_{n}^{2}-(1-q) \gamma_{n} \delta_{n} \geq 0$

Remark 2. i) If $\sigma=\tau=\theta=\eta=0$ then $Q H$ with these parameters (defined e.g. by polynomials $p_{n}$ given by (1.7)) is $q$-Wiener process as described in $[12$ and [5].

ii) If $\sigma=\tau=\eta=0$, and $q=\theta=1$ then $Q H$ with these parameters (defined e.g. by polynomials $p_{n}$ given by (1.7)) is centered Poisson process as described in [5. This statement is easier to verify if one returns to variable $x \longrightarrow y \sqrt{t}$ which would result in the following 3-term recurrence:

$$
x \tilde{M}_{n}(x)=\tilde{M}_{n+1}(x)+n \tilde{M}_{n}(x)+n \tilde{M}_{n-1}(x),
$$

where we denoted $\tilde{M}_{n}(x)=M_{n}(y \sqrt{t})$.

iii) If we define generalized Chebyshev polynomials by $C_{n}(x)=T_{n}((x-a) / b)$ then we see that polynomials $C_{n}$ satisfy the following 3-term recurrence:

$$
x C_{n}(x)=C_{n+1}(x)+a C_{n}(x)+b^{2} C_{n-1}(x) .
$$

Comparing this formula with the ones given in assertions vi) and vii) of Lemma 2 we deduce that respective processes are compactly supported and we are able to describe completely their 1-dimensional distributions. 


\section{REMARKS AND OPEN PROBLEMS}

(1) We have solved system of recurrences (1.1)-(1.6) for some special values of parameters. Of course it would be interesting to find general solution i.e. for all sensible values of parameters. By sensible values we mean those assuring non-negativity of the sequence $\chi_{n}$ since then the measure that makes polynomials $M_{n}$ orthogonal is positive i.e. probabilistic. Sensible might mean also that sequences $\gamma_{n}, \delta_{n}$ and $\chi_{n}$ are bounded since then the probability measure would be compactly supported.

(2) First of all notice that the set of allowed values of parameters $\sigma, \tau, \theta$, $\eta, q$ is such that $\sigma, \tau \geq 0, \theta, \eta \in \mathbb{R}$ and $q \leq 1+2 \sqrt{\sigma \tau}$ as pointed out in [5]. As it follows from the assertion iv) of Proposition 3 below if $q \in$ $\left(1-2 \sqrt{\sigma \tau}, 1+2 \sqrt{\sigma \tau}\right.$ the sequence $\left\{\lambda_{n}\right\}$ changes sign infinitely often. Hence it is rather unlikely that a set of OMP defining positive 1 - dimensional measure exists. Is it really so? Do there exist QH that are not Markovian and $q>1-2 \sqrt{\sigma \tau}$ ?

(3) It is not sure if parameters satisfying $\sigma, \tau \geq 0, \theta, \eta \in \mathbb{R}$ and $q \leq 1+2 \sqrt{\sigma \tau}$ or even $q \leq 1-2 \sqrt{\sigma \tau}$ can guarantee that the sequence $\left\{\chi_{n}\right\}$ defined by (2.6) is non-negative. It might not be true in general since expression

$$
1+\theta \gamma_{n}+\tau \gamma_{n}^{2}+\eta \delta_{n}+\sigma \delta_{n}^{2}-(1-q) \gamma_{n} \delta_{n}
$$

can be written as a quadratic form in $(\theta, \eta)$ as pointed out in Corollary 1 . However matrix of this form might not be positive definite. This is so since matrix $\boldsymbol{\Delta}$ defined there is non-positive definite hence, at least theoretically the values of this expression can be negative and this may result in negative values of some elements of the sequence $\left\{\chi_{n}\right\}$. Relatively simple case occurs when $\theta=\eta=0$. Then as we have shown if $q+\sigma \tau \geq 0$ then sequence $\left\{\chi_{n}\right\}$ is positive and bounded. If $q+\sigma \tau<0$ many numerical simulations show that also in this case the sequence $\left\{\chi_{n}\right\}$ is positive. But proving it we leave to more gifted researchers.

(4) The set of OMP of a given QH supplies knowledge about 1-dimensional distributions. But in fact knowing polynomials of OMP $\left\{p_{j}\right\}$ we can also state something about transitional probability distribution. Namely from the relationship (A.7) we can also deduce that orthogonal polynomials $\left\{W_{n}\right\}$ of the transitional probability must be of the form :

$$
W_{n}\left(X_{t}, t ; X_{s}, s\right)=\sum_{j=0}^{n} V_{n, j}\left(X_{s}, s\right)\left(p_{j}\left(X_{t}, t\right)-p_{j}\left(X_{s}, s\right)\right),
$$

for $s \leq t$ and some polynomials $V_{n, j}\left(X_{s}, s\right)$ of order at most $n-j$. This is so since we have $E_{x} W_{n}\left(X_{t}, t ; x, s\right)=0$ for all $n \geq 1$. Thus it remains to prove that these polynomials satisfy some 3 -term recurrence to be able to identify them as polynomials orthogonal with respect to the transitional measure. It would be be interesting to find this 3-term recurrence. The examples known so far suggest to seek polynomials $V_{n, j}\left(X_{s}, s\right)$ among such polynomials that :

$$
\sum_{j=0}^{n} V_{n-j}(x ; s) p_{j}(x, s)=0
$$


for a set of polynomials that are of order $n-j$ and indexed only one integer index. Can it be true in the general case?

(5) Finally notice that in Proposition 2 we did not consider cases $(\eta, \theta)=(q, \eta)$ $=(q, \theta)=(0,0)$. Together with 7 cases considered in assertions i)-iv) of this Proposition these 3 cases would make $10=\left(\begin{array}{l}5 \\ 2\end{array}\right)$ cases of 2 out of 5 parameters $(\sigma, \tau, \theta, \eta, q)$ that are set to zero. The cases $(\eta, \theta)=(q, \eta)=$ $(q, \theta)=(0,0)$ were not considered because they are too complicated and seem to require deeper, more detailed analysis. We leave them to more talented researchers.

\section{Auxiliary Results}

In this section we will analyze properties of the sequence $\left\{\lambda_{n}\right\}$ that appear when examining properties of marginal distributions of MQH. As the considerations presented above show it plays a crucial rôle in the whole analysis.

One can easily observe that right hand side of (2.1) defines a transformation of $\lambda_{n}$ which is known under the name of Möbius transform. Much is known about its properties. However we need only those properties of it that fit to our special setting. Thus it seems to be easier and more logical to prove these needed properties once more than to dig in the literature and reduce general cases to our setting. All the more the proofs in these special cases are elementary although not quite simple.

Proposition 3. Let us denote $f(x \mid q, z)=\frac{1+q x}{1-z x}$ and assume $z \geq 0$.

i) If $q+z \geq 0$ and $x<1 / z$ then $f(x \mid q, z) \geq x \geq 0$. In particular if $q+z=0$ then $f(x \mid q, z)=1$, if $q+z<0$ then for $0 \leq x \leq \frac{-1}{q}, 0 \leq f(x \mid q, z) \leq x$. In particular if $q=-1$ we have $f(0 \mid q, z)=1$ and $f(1 \mid q, z)=0$.

ii) If $z \in[0,1)$ and $q \in[-1,1-2 \sqrt{z}]$ then for $x \in\left[0, \frac{1}{\sqrt{z}}\right)$ implies that $f(x \mid q, z) \in$ $\left[0, \frac{1}{\sqrt{z}}\right)$.

iii) Let $f^{(n)}$ denote $n$ - fold composition of function $f$. If $q \leq 1-2 \sqrt{z}$ or $q \geq$ $1+2 \sqrt{z}$ then for every $n$ there exists a number $y_{n}$ such that $y_{n}=f^{(n)}\left(y_{n} \mid q, z\right)$. Otherwise such number does not exist. Moreover if they do exist all numbers $y_{n}$, are identical and equal to

$$
y(q, z)=\frac{2}{1-q+\sqrt{(1-q)^{2}-4 z}} .
$$

iv) If $z \in[0,1)$ and $q \in[-1,1-2 \sqrt{z})$ and $x \in\left[0, \frac{1}{\sqrt{z}}\right)$ if $q+z \geq 0$ and $x \in\left[0, \frac{1}{|q|}\right)$ when $q+z<0$ we have:

$$
|f(x \mid q, z)-y(q, z)|<C(q, z)|x-y(q, z)|,
$$

with $C(q, z)<1$.

v) If $q>1-2 \sqrt{z}$ then sequence $\left\{\lambda_{n}\right\}$ changes sign infinitely many times.

Proof. First of all notice that $f(x \mid q, z)=1+\frac{(q+z) x}{1-z x}$ and $f^{\prime}(x \mid q, z)=\frac{q+z}{(1-z x)^{2}}$. i) If $q+z \geq 0$ then the derivative of the function $f(x)$ is nonnegative. If $q+z=0$ we see that $f(x \mid q, z)-1=0$. If $q+z<0$ analyzing polynomial $(1+q x)(1-z x)$ we see that function $f(x \mid q, z)$ is decreasing and nonnegative on $0 \leq x \leq \frac{-1}{q}$ hence the first assertion is true.

ii) If $q+z<0$ then we have for $\frac{1}{\sqrt{z}}>x \geq 0$ we have $f(x)=1+\frac{(q+z) x}{(1-x z)}<1 \leq \frac{1}{\sqrt{z}}$. If $q+z=0$ then $f(x)=1<\frac{1}{\sqrt{z}}$. If $q+z>0$ which is equivalent in this case to 
$0<(q+z) \leq(1-\sqrt{z})^{2}$ then $f(x)$ is increasing and we have $f(x)<f\left(\frac{1}{\sqrt{z}}\right)=1+$ $\frac{(q+z)}{\sqrt{z}(1-\sqrt{z})} \leq 1+\frac{1-\sqrt{z}}{\sqrt{z}}=\frac{1}{\sqrt{z}}$.

iii) If $z=0$ then $f(x)=1+q x$, hence $f^{(n)}(x)=[n-1]_{q}+q^{n} x$, consequently $y_{n}=\frac{1}{1-q}$. Assume that $z \neq 0$. Let us notice that

$$
f^{(n)}(x)=\frac{A_{n}+B_{n} x}{C_{n}-D_{n} x}
$$

for some depending on $q$ and $z$ functions $A_{n}, B_{n}, C_{n}, D_{n}$. Notice that the solution of the equation $f^{(n)}\left(y_{n}\right)=y_{n}$ satisfies the quadratic equation:

$$
D_{n} y^{2}+\left(B_{n}-C_{n}\right) y+A_{n}=0 .
$$

Since $f\left(f^{(n)}(x)\right)=f^{(n)}(f(x))$ for every $x$ we deduce that:

$$
\begin{aligned}
A_{n}+B_{n} & =q A_{n}+C_{n}, \\
-z A_{n}+q B_{n} & =q B_{n}-D_{n} \\
C_{n}-D_{n} & =C_{n}-z A_{n}, \\
C_{n} z+q D_{n} & =D_{n}+z B_{n},
\end{aligned}
$$

and consequently that $B_{n}-C_{n}=(1-q) A_{n}$ and $D_{n}=z A_{n}$. Since $A_{n} \neq 0$ (otherwise we would have $\left.f^{(n)}(x) \equiv x\right)$ we deduce that for all $n$ number $y_{n}$ satisfies equation

$$
z y^{2}+(1-q) y+1=0 .
$$

Moreover real solution of this equation exists if $(1-q)^{2} \geq 4 z$ or equivalently if $q \leq 1-2 \sqrt{z}$ or $q \geq 1+2 \sqrt{z}$. Now let us consider the case $q \in(1-2 \sqrt{z}, 1+2 \sqrt{z})$. Then as the above analysis shows there is no solution of the equation $f^{(n)}(x)=x$ for any $n>0$.

iv) First of all recall $-1+z \leq q+z \leq(1-\sqrt{z})^{2}$ and that $y(q, z)=\frac{1+q y(q, z)}{1-z y(q, z)}$. Now assume that $q+z>0$ then

$$
|f(x \mid q, z)-y(q, z)|=\frac{|x-y(q, z)||q+z|}{(1-z x)(1-z y(q, z) \mid} \leq C(q, z)|x-y(q, z)|,
$$

where $C(q, z)=\frac{\mid q+z) \mid}{(1-\sqrt{z})^{2}}<1$ since $y(q, z) \leq \frac{2}{1-q} \leq \frac{1}{\sqrt{z}}$. If $q+z=0$ then $f(x \mid q, z)$ $=y(-z, z)=1$. So it remains to consider the case $0>q+z>z-1$. Recall that function $f$ is now decreasing and thus for $0 \leq x \leq \frac{-1}{q}$ we have:

$$
\begin{aligned}
|f(x \mid q, z)-y(q, z)| & =\frac{|x-y(q, z)||q+z|}{(1-z x)(1-z y(q, z))} \leq \frac{|x-y(q, z)||q+z|}{\left|\frac{q+z}{q}\right|(1-z y(q, z))} \\
& \leq|x-y(q, z)| C(q, z) .
\end{aligned}
$$

where $C(q, z)=\frac{|q|}{(1-z y(q, z))}=|q| \frac{1+q y(q, z)}{y(q, z)}=|q|\left|\frac{1}{y(q, z)}+q\right|=$ $|q|\left|q+\frac{1}{2}-\frac{1}{2} q-\frac{1}{2} \sqrt{(1-q)^{2}-4 z}\right| \leq|q| \max \left(\left|\frac{1+q}{2}\right|, \frac{(1-q)}{2} \mid<1\right.$.

v) If $q>1-2 \sqrt{z}$, then as it follows from assertion iii) of Proposition 3 there is no condensation point of the sequence $\left\{\lambda_{n}\right\}$. Since in this case we have $q+z \geq 0$ then the sequence $\left\{\lambda_{n}\right\}$ is increasing and consequently will will reach value more that $\frac{1}{z}$. But then the next iterate will be negative and again the sequence will be increasing and so on. 


\section{Proofs}

Proof of Lemma 1, i) We base on Lemma 3 with $z=\sigma \tau$ and $q \in(-1,1-2 \sqrt{\sigma \tau})$. For $q=1-2 \sqrt{\sigma \tau}$ see the assertion vi) of Proposition 2 ,

ii) Notice that $\operatorname{det} A_{n}=\left(1-\sigma \tau \lambda_{n}\right)^{2}-\sigma \tau\left(1+q \lambda_{n}\right)^{2}$. If $\operatorname{det} A_{n}=0$ then we would have $\lambda_{n+1}^{2}=\frac{1}{\sigma \tau}$ which cannot happen since assertion ii) of Proposition 2 states that if $\lambda_{n} \in\left[0, \frac{1}{\sqrt{\sigma \tau}}\right)$ then also $\lambda_{n+1} \in\left[0, \frac{1}{\sqrt{\sigma \tau}}\right)$ and we start from $\lambda_{0}=0$.

iii) We have $\left(1-\sigma \tau\left(2 \lambda_{n}+q \lambda_{n}^{2}\right)\right)=\left(1-\sigma \tau \lambda_{n}\right)^{2}-\sigma \tau(\sigma \tau+q) \lambda_{n}^{2}$. hence $(1-$ $\left.\sigma \tau\left(2 \lambda_{n}+q \lambda_{n}^{2}\right)\right) \geq 0$ is equivalent to $1 \geq(\sqrt{\sigma \tau}+\sqrt{q+\sigma \tau}) \sqrt{\sigma \tau} \lambda_{n}$. Now keeping in mind that $\sqrt{\sigma \tau} \lambda_{n} \leq 1$ and $\sqrt{\sigma \tau}+\sqrt{q+\sigma \tau} \leq 1$ for $q \leq 1-2 \sqrt{\sigma \tau}$ we see that it is true. Now if $q+\sigma \tau \geq 0$ then $\left(q+\sigma \tau-\sigma \tau\left(1-\lambda_{n-1}\right)^{2}\right) \geq 0$ is equivalent to $\sqrt{q+\sigma \tau} \geq \sqrt{\sigma \tau}\left(\lambda_{n}-1\right)$. But $\lambda_{n}-1=\frac{(q+\sigma \tau) \lambda_{n-1}}{1-\sigma \tau \lambda_{n-1}}$. So the last inequality is true if $1-\sigma \tau \lambda_{n-1} \geq \sqrt{\sigma \tau} \sqrt{q+\sigma \tau} \lambda_{n-1}$ which is true by the previous argument.

iv) Denote $\sigma \tau$ as $z$ for simplicity. We will write $y$ instead of $y(q, z)$ for simplicity. First of all notice that we have: $y-y^{2} z=1+q y$, so $z y^{2}=y(1-q)-1$. Next we have $q+2 z \lambda_{n}-z \lambda_{n}^{2} \longrightarrow q+2 z y-y(q-1)+1=1+q+2 z y-y(1-q)$ and $\left(1-z\left(2 \lambda_{n}+q \lambda_{n}^{2}\right)\right) \longrightarrow 1-2 z y-q(y(1-q)-1)=1+q-2 z y-q y(1-q)$ by assertion iv) of Lemma 3. Now keeping in mind that $\frac{1}{y(q, z)}=\left(1-q+\sqrt{(1-q)^{2}-4 z}\right) / 2$ we get:

$$
\begin{gathered}
D(q, \sigma \tau)=\frac{1+q+2 z y-y(1-q)}{1+q-2 z y-q y(1-q)} \\
=\frac{(1+q) \sqrt{(1-q)^{2}-4 z}+4 z+2 q-2+1-q^{2}}{(1+q) \sqrt{(1-q)^{2}-4 z}+1-q^{2}-4 z-2 q+2 q^{2}} \\
=\frac{1+q-\sqrt{(1-q)^{2}-4 z}}{1+q+\sqrt{(1-q)^{2}-4 z}} .
\end{gathered}
$$

Then we have on one side: $1+q-\sqrt{(1-q)^{2}-4 z}<1+q+\sqrt{(1-q)^{2}-4 z}$ for $4 z<(1-q)^{2}$ and on the other side $1+q-\sqrt{(1-q)^{2}-4 z}>-\left(1+q+\sqrt{(1-q)^{2}-4 z}\right)$ which is also true for $q>-1$.

v) Obviously sequence $\left\{\chi_{n}\right\}$ is defined by the recursion

$$
\chi_{n+1}=\kappa_{n} \chi_{n}+\frac{1}{\left(1-\sigma \tau\left(2 \lambda_{n}+q \lambda_{n}^{2}\right)\right)},
$$

where we denoted $\kappa_{n}=\frac{\left(q+\sigma \tau-\sigma \tau\left(1-\lambda_{n-1}\right)^{2}\right)}{\left(1-\sigma \tau\left(2 \lambda_{n}+q \lambda_{n}^{2}\right)\right)}$. Next we notice that: (a) (5.1) is a inhomogeneous linear recursive equation of the first degree, (b) as shown above sequences $\left\{\kappa_{n}\right\}_{n \geq 1},\left\{\frac{1}{\left(1-\sigma \tau\left(2 \lambda_{n}+q \lambda_{n}^{2}\right)\right)}\right\}_{n \geq 1}$ have limits and moreover the limit of $\left\{\kappa_{n}\right\}$ has absolute value less than 1 . Now by standard argument used in the case of linear recursions we deduce that $\chi_{n}$ has a limit $\xi$ and moreover this limit satisfies equation

$$
\xi=D(q, \sigma \tau) \xi+\frac{1-q+\sqrt{(1-q)^{2}-4 z}}{\sqrt{(1-q)^{2}-4 z}\left((1+q)+\sqrt{(1-q)^{2}-4 z}\right)} .
$$


So

$$
\begin{gathered}
\xi=\frac{1-q+\sqrt{(1-q)^{2}-4 z}}{\sqrt{(1-q)^{2}-4 z}\left((1+q)+\sqrt{(1-q)^{2}-4 z}\right)} /(1-D(q, \sigma \tau)) \\
=\frac{1-q+\sqrt{(1-q)^{2}-4 z}}{\sqrt{(1-q)^{2}-4 z}\left((1+q)+\sqrt{(1-q)^{2}-4 z}\right)} \frac{1+q+\sqrt{(1-q)^{2}-4 \sigma \tau}}{2 \sqrt{(1-q)^{2}-4 \sigma \tau}} \\
=\frac{\left(1-q+\sqrt{(1-q)^{2}-4 \sigma \tau}\right)}{2 \sqrt{(1-q)^{2}-4 \sigma \tau}} .
\end{gathered}
$$

To see that $\chi_{n} \geq 0$ for all $n \geq 1$ we note that above we have shown that if $q+\sigma \tau \geq 0$ the $\kappa_{n} \geq 0$.

Proof of Proposition 2. First of all notice that if $\sigma \tau=0$ then $\lambda_{n}=[n]_{q}$ since then equation (2.1) reduces to $\lambda_{n+1}=q \lambda_{n}+1$, with $\lambda_{0}=0$.

i) $\tau=\theta=0$ Under our assumptions we get $A_{n}=\left[\begin{array}{cc}1 & -\sigma[n+1]_{q} \\ 0 & 1\end{array}\right], B_{n}=$ $\left[\begin{array}{cc}q & -\sigma q[n-1]_{q} \\ 0 & q\end{array}\right]$ and $C_{n}=\left[\begin{array}{cc}\sigma[n]_{q} & 1 \\ 1 & 0\end{array}\right]$ since $1+q[n]_{q}=[n+1]_{q}$ and $1-[n]_{q}=$ $-q[n-1]_{q}$. So $A_{n}^{-1} B_{n}=\left[\begin{array}{cc}q & \sigma(1+q) q^{n} \\ 0 & q\end{array}\right]$ and $A_{n}^{-1} C_{n}\left[\begin{array}{l}0 \\ \eta\end{array}\right]=\left[\begin{array}{l}\eta \\ 0\end{array}\right]$. Hence vector $\left[\begin{array}{l}\gamma_{n} \\ \delta_{n}\end{array}\right]$ satisfies the following recursion: $\left[\begin{array}{c}\gamma_{n+1} \\ \delta_{n+1}\end{array}\right]=\left[\begin{array}{cc}q & \sigma(1+q) q^{n} \\ 0 & q\end{array}\right]\left[\begin{array}{c}\gamma_{n} \\ \delta_{n}\end{array}\right]$ $+\left[\begin{array}{l}\eta \\ 0\end{array}\right]$. So $\delta_{n}=0$, while $\gamma_{n}=[n]_{q} \eta$. Further we have

$1+\theta \gamma_{n}+\tau \gamma_{n}^{2}+\eta \delta_{n}+\sigma \delta_{n}^{2}-\left.(1-q) \gamma_{n} \delta_{n}\right|_{\tau=0, \theta=0, \gamma_{n}=\eta[n]_{q}, \delta_{n}=0}=1$, so recursion (2.6) reduces to

$$
q \chi_{n}+1=\chi_{n+1},
$$

with $\chi_{1}=1$. Thus indeed $\chi_{n}=[n]_{q}$. If $\sigma=\eta=0$ we have symmetric situation.

ii) $\tau=\eta=0$. We get then $A_{n}=\left[\begin{array}{cc}1 & -\sigma q[n+1]_{q} \\ 0 & 1\end{array}\right], B_{n}=\left[\begin{array}{cc}q & -\sigma q[n-1]_{q} \\ 0 & q\end{array}\right]$ and $C_{n}=\left[\begin{array}{cc}\sigma[n]_{q} & 1 \\ 1 & 0\end{array}\right]$. Hence $A_{n}^{-1} B_{n}=\left[\begin{array}{cc}q & \sigma(1+q) q^{n} \\ 0 & q\end{array}\right]$ and $A_{n}^{-1} C_{n}\left[\begin{array}{l}\theta \\ 0\end{array}\right]=$ $\left[\begin{array}{c}\sigma \theta\left(q^{n}+2[n]_{q}\right) \\ 0\end{array}\right]$. Hence vector $\left[\begin{array}{c}\gamma_{n} \\ \delta_{n}\end{array}\right]$ satisfies the following recursion: $\left[\begin{array}{c}\gamma_{n+1} \\ \delta_{n+1}\end{array}\right]$ $=\left[\begin{array}{cc}q & \sigma(1+q) q^{n} \\ 0 & q\end{array}\right]\left[\begin{array}{l}\gamma_{n} \\ \delta_{n}\end{array}\right]+\left[\begin{array}{c}\sigma \theta\left(q^{n}+2[n]_{q}\right) \\ \theta\end{array}\right]$. So $\delta_{n}=[n]_{q} \theta$ and sequence $\gamma_{n}$ satisfies recursion:

$$
\gamma_{n+1}=q \gamma_{n}+\sigma \theta(1+q) q^{n}[n]_{q}+\sigma \theta\left(q^{n}+2[n]_{q}\right) .
$$

One can easily check that

$$
\begin{aligned}
& {[n+1]_{q}\left(q^{n}+2[n]_{q}\right)-q[n]_{q}\left(q^{n-1}+2[n-1]_{q}\right) } \\
= & q^{n}(1+q)[n]_{q}+q^{n}+2[n]_{q},
\end{aligned}
$$

since we have $[n+1]_{q}\left(q^{n}+2[n]_{q}\right)-q[n]_{q}\left(-q^{n-1}+2[n]_{q}\right)=2[n]_{q}\left([n+1]_{q}-q[n]_{q}\right)$ $+q^{n}[n+1]_{q}+q^{n}[n]_{q}=2[n]_{q}+q^{n}+q^{n}[n]_{q}+q^{n}\left([n+1]_{q}-1\right]=[n]_{q}+[n+1]_{q}+$ $q^{n}(1+q)[n]_{q}$. Hence we deduce that $\gamma_{n}=[n]_{q}\left([n]_{q}+[n-1]_{q}\right) \theta \sigma$ by direct checking 
and uniqueness of the solution. Using these results we can write recursion to be satisfied by sequence $\chi_{n}$ :

$$
\begin{aligned}
\chi_{n+1} & =q \chi_{n}+1+\theta^{2} \sigma[n]_{q}\left([n]_{q}+[n-1]_{q}\right)+\sigma \theta^{2}[n]_{q}^{2}-(1-q) \theta^{2} \sigma[n]_{q}^{2}\left([n]_{q}+[n-1]_{q}\right) \\
& =q \chi_{n}+1+\theta^{2} \sigma[n]_{q}\left(2[n]_{q}+[n-1]_{q}-\left(1-q^{n}\right)\left([n]_{q}+[n-1]_{q}\right)\right) \\
& =q \chi_{n}+1+\theta^{2} \sigma[n]_{q}\left([n]_{q}+q^{n}\left([n]_{q}+[n-1]_{q}\right)\right) .
\end{aligned}
$$

Let us denote $\zeta_{n}=\chi_{n}-[n]_{q}$. We see that sequence $\zeta_{n}$ satisfies the following recursion:

$$
\zeta_{n+1}=q \zeta_{n}+\theta^{2} \sigma[n]_{q}\left([n]_{q}+q^{n}\left([n]_{q}+[n-1]_{q}\right)\right)
$$

Let us notice that

$$
\begin{aligned}
& {[n+1]_{q}[n]_{q}^{2}-q[n]_{q}[n-1]_{q}^{2} } \\
= & {[n]_{q}\left([n]_{q}+q q^{n-1}\left([n]_{q}+[n-1]_{q}\right),\right.}
\end{aligned}
$$

since $[n]_{q}\left([n+1]_{q}[n]_{q}-q[n-1]_{q}^{2}\right)=[n]_{q}\left([n]_{q}+q[n]_{q}^{2}-q[n-1]_{q}^{2}\right)$. Hence we see that $\zeta_{n}=[n]_{q}[n-1]_{q}^{2} \theta^{2} \sigma$. Similarly we show the other statement of this assertion.

iii) Under our assumptions we have $A_{n}=I, B_{n}=\left[\begin{array}{ll}q & 0 \\ 0 & q\end{array}\right], C_{n}=\left[\begin{array}{ll}0 & 1 \\ 1 & 0\end{array}\right]$. Vector $\left[\begin{array}{l}\gamma_{n} \\ \delta_{n}\end{array}\right]$ in fact satisfies two separate equations : $\gamma_{n+1}=q \gamma_{n}+\eta$ which results in $\gamma_{n}=\eta[n]_{q}$ and $\delta_{n+1}=q \delta_{n}+\theta$. Which results in $[n]_{q} \theta$. Now Inserting these quantities to equation (2.6) yields the following recursion:

$$
\chi_{n+1}=q \chi_{n}+1+\theta \eta[n]_{q}\left(1+q^{n}\right) \text {. }
$$

Again we have

$$
\begin{aligned}
& {[n+1]_{q}[n]_{q}-q[n]_{q}[n-1]_{q} } \\
= & {[n]_{q}\left(1+q^{n}\right) . }
\end{aligned}
$$

Thus we deduce that $\chi_{n}=[n]_{q}+\theta \eta[n]_{q}[n-1]_{q}$.

iv) Assumption that $\sigma=0$ implies that $\lambda_{n}=1$. Thus we have $A_{n}=\left[\begin{array}{cc}1 & 0 \\ -\tau & 1\end{array}\right]$, $B_{n}=\left[\begin{array}{ll}0 & 0 \\ 0 & 0\end{array}\right], C_{n}=\left[\begin{array}{ll}0 & 1 \\ 1 & \tau\end{array}\right]$. Hence $\gamma_{n}$ and $\delta_{n}$ do not depend on $n$ and it is elementary that they are equal to $\eta$ and $\theta+2 \eta \tau$ respectively. Further we have $\left.\left(q+\sigma \tau-\sigma \tau\left(1-\lambda_{n}\right)^{2}\right)\right|_{\sigma=0, q=0}=0$ and $\left.\left(1-\sigma \tau\left(2 \lambda_{n}+q \lambda_{n}^{2}\right)\right)\right|_{\sigma=0, q=0}=1$ and $\theta \gamma_{n}$ $+\eta \delta_{n}+\tau \gamma_{n}^{2}+\sigma \delta_{n}^{2}-(1-q) \gamma_{n} \delta_{n}+1=1+\theta \eta+\tau \eta^{2}$. In case $\tau=q=1$ we argue in the similar way.

v) Under this assumption equation (2.1) reduces to

$$
\lambda_{n+1}=\frac{1-\sigma \tau \lambda_{n}}{1-\sigma \tau \lambda_{n}}=1 \text {. }
$$

Besides we have for $n \geq 1: A_{n}=\left[\begin{array}{cc}1-\tau \sigma & -\sigma(1-\sigma \tau) \\ -\tau(1-\sigma \tau) & 1-\sigma \tau\end{array}\right], B_{n}=\left[\begin{array}{ll}0 & 0 \\ 0 & 0\end{array}\right]$, $C_{n}=C_{n}=\left[\begin{array}{ll}\sigma & 1 \\ 1 & \tau\end{array}\right]$, so $\Xi_{n}=A_{n}^{-1} B_{n}=\left[\begin{array}{ll}0 & 0 \\ 0 & 0\end{array}\right] \stackrel{d f}{=} \Xi$. Further $\prod_{k=1}^{n} \Xi_{k}=0, w_{n}$ $=\frac{1}{(1-\sigma \tau)^{2}}\left[\begin{array}{c}2 \theta \sigma+\eta(1+\sigma \tau) \\ 2 \eta \tau+\theta(1+\sigma \tau)\end{array}\right]$. So $\left[\begin{array}{l}\gamma_{n} \\ \delta_{n}\end{array}\right]=\frac{1}{(1-\sigma \tau)^{2}}\left[\begin{array}{c}2 \theta \sigma+\eta(1+\sigma \tau) \\ 2 \eta \tau+\theta(1+\sigma \tau)\end{array}\right]$ for $n \geq$ 0. Besides $\left.\left(q+\sigma \tau-\sigma \tau\left(1-\lambda_{n}\right)^{2}\right)\right|_{q+\sigma \tau=0}=0$ and $\left.\left(1-\sigma \tau\left(2 \lambda_{n}+q \lambda_{n}^{2}\right)\right)\right|_{q+\sigma \tau=0}$ 
$=(1-\sigma \tau)^{2}$. Since $\gamma_{n}$ and $\delta_{n}$ do not depend on $n$ and we have: $\theta \gamma_{n}+\eta \delta_{n}=$ $\frac{2(\theta+\tau \eta)(\eta+\theta \sigma)}{(1-\sigma \tau)^{2}}$ and $\tau \gamma_{n}^{2}+\sigma \delta_{n}^{2}-(1-q) \gamma_{n} \delta_{n}=\frac{-(\theta+\tau \eta)(\eta+\theta \sigma)}{(1-\sigma \tau)^{2}}$. Hence we deduce we deduce that $\beta_{n-1} \varepsilon_{n}$ also does not depend on $n$. By direct calculation we have $\chi_{1}$ $=\frac{1}{(1-\sigma \tau)^{2}}$, while for $n>1$ we have: $1+\theta \gamma_{n}+\eta \delta_{n}+\tau \gamma_{n}^{2}+\sigma \delta_{n}-(1-q) \gamma_{n} \delta_{n}=1$ $+\frac{(\theta+\tau \eta)(\eta+\theta \sigma)}{(1-\sigma \tau)^{2}}$.

vi) First of all notice that under our assumptions we have $q+\sigma \tau=(1-\sqrt{\sigma \tau})^{2}$. Next notice that if $n=1$ then $\lambda_{1}=1=\left.\frac{n}{1+(n-1) \sqrt{\sigma \tau}}\right|_{n=1}$. Hence by induction we have $1+(1-2 \sqrt{\sigma \tau}) n /(1+(n-1) \sqrt{\sigma \tau}))=\frac{(1-\sqrt{\sigma \tau})(n+1)}{1+(n-1) \sqrt{\sigma \tau}}$ and $1-\sigma \tau n /(1+(n-1) \sqrt{\sigma \tau})$ $=\frac{(1-\sqrt{\sigma \tau})(1+n \sqrt{\sigma \tau})}{(1+(n-1) \sqrt{\sigma \tau})}$. Thus

$$
\lambda_{n+1}=\left(1+q \lambda_{n}\right) /\left.\left(1-\sigma \tau \lambda_{n}\right)\right|_{\lambda_{n}=n /(1+(n-1) \sqrt{\sigma \tau})}=\frac{n+1}{1+n \sqrt{\sigma \tau}} .
$$

Now notice that $q+\sigma \tau-\left.\sigma \tau\left(1-\lambda_{n-1}\right)^{2}\right|_{q=1-2 \sqrt{\sigma \tau}}=(1-\sqrt{\sigma \tau})^{2}-\sigma \tau\left(1-\frac{n-1}{1+(n-2) \sqrt{\sigma \tau}}\right)^{2}$ $=\frac{(1-\sqrt{\sigma \tau})^{2}(1+2(n-2) \sqrt{\sigma \tau})}{(1+(n-2) \sqrt{\sigma \tau})^{2}}$ and $\left.\left(1-\sigma \tau\left(2 \lambda_{n}+q \lambda_{n}^{2}\right)\right)\right|_{q=1-2 \sqrt{\sigma \tau}}=\frac{(1-\sqrt{\sigma \tau})^{2}(1+2 n \sqrt{\sigma \tau})}{(1+(n-1) \sqrt{\sigma \tau})^{2}}$. So sequence $\left\{\chi_{n}\right\}$ satisfies the following recursion:

$$
\chi_{n+1}=\frac{(1+2(n-2) \sqrt{\sigma \tau})(1+(n-1) \sqrt{\sigma \tau})^{2}}{(1+2 n \sqrt{\sigma \tau})(1+(n-2) \sqrt{\sigma \tau})^{2}} \chi_{n}+\frac{(1+(n-1) \sqrt{\sigma \tau})^{2}}{(1-\sqrt{\sigma \tau})^{2}(1+2 n \sqrt{\sigma \tau})} .
$$

The proof is now by induction setting $n=1$ in (2.10) we get 1 . Further by direct calculation we have:

$$
\begin{gathered}
\frac{n(1+(n-2) \sqrt{\sigma \tau})^{2}(1+(n-3) \sqrt{\sigma \tau})}{(1-\sqrt{\sigma \tau})^{2}(1+2(n-1) \sqrt{\sigma \tau})(1+2(n-2) \sqrt{\sigma \tau})} \times \frac{(1+2(n-2) \sqrt{\sigma \tau})(1+(n-1) \sqrt{\sigma \tau})^{2}}{(1+2 n \sqrt{\sigma \tau})(1+(n-2) \sqrt{\sigma \tau})^{2}} \\
+\frac{(1+(n-1) \sqrt{\sigma \tau})^{2}}{(1-\sqrt{\sigma \tau})^{2}(1+2 n \sqrt{\sigma \tau})}=\frac{(n+1)(1+(n-1) \sqrt{\sigma \tau})^{2}(1+(n-2) \sqrt{\sigma \tau})}{(1-\sqrt{\sigma \tau})^{2}(1+2 n \sqrt{\sigma \tau})(1+2(n-1) \sqrt{\sigma \tau})} .
\end{gathered}
$$

\section{REFERENCES}

[1] Akhiezer, N. I. The classical moment problem and some related questions in analysis. Translated by N. Kemmer Hafner Publishing Co., New York 1965 x+253 pp. MR0184042 (32 \#1518)

[2] Bryc, W. , Wesołowski, J. (2005), Conditional Moments of $q$-Meixner Processes, Probab. Theory Rel. Fields 131, 415-441

[3] Bryc, W., Wesołowski, J. (2007), Bi - Poissson process, Infinite Dimensional Analysis, Quantum Probability and Related Topics 10 (2) , 277-291

[4] Bryc, W. , Matysiak, W. , Wesołowski, J., The Bi - Poisson process: a quadratic harness. Annals of Probability 36 (2) (2008), s. 623-646

[5] Wlodzimierz Bryc, Wojciech Matysiak, Jacek Wesolowski. Quadratic Harnesses, $q$-commutations, and orthogonal martingale polynomials. Trans. Amer. Math. Soc. 359 (2007), no. 11, 5449-5483

[6] Włodek Bryc, Jacek Wesołowski. Askey-Wilson polynomials, quadratic harnesses and martingales. IMS-AOP-AOP503. Annals of Probability 2010, Vol. 38, No. 3, 1221-1262.

[7] Wlodzimierz Bryc, Wojciech Matysiak, Jacek Wesołowski, Free Quadratic Harness. Stochastic Processes and their Applications 121 (2011) 657-671

[8] Bryc, Włodzimierz, Wesołowski Jacek, Stitching pairs of Levy processes into harnesses, Stochastic Processes and their Applications, 122(2012), 2854-2869

[9] Ismail, Mourad E. H. Classical and quantum orthogonal polynomials in one variable. With two chapters by Walter Van Assche. With a foreword by Richard A. Askey. Encyclopedia 
of Mathematics and its Applications, 98. Cambridge University Press, Cambridge, 2005. xviii+706 pp. ISBN: 978-0-521-78201-2; 0-521-78201-5 MR2191786 (2007f:33001)

[10] Koekoek, Roelof; Lesky, Peter A.; Swarttouw, René F. Hypergeometric orthogonal polynomials and their $\$ \mathrm{q} \$$-analogues. With a foreword by Tom H. Koornwinder. Springer Monographs in Mathematics. Springer-Verlag, Berlin, 2010. xx+578 pp. ISBN: 978-3-642-05013-8 MR2656096 (2011e:33029)

[11] Simon, Barry. The classical moment problem as a self-adjoint finite difference operator. Adv. Math. 137 (1998), no. 1, 82-203. MR1627806 (2001e:47020)

[12] Szabłowski, Paweł J. $q-$ Wiener and $(\alpha, q)$ - Ornstein-Uhlenbeck processes. A generalization of known processes, Theory of Probability and Its Applications, 56 (4), 2011, 742-772, http://arxiv.org/abs/math/0507303

\section{Appendix A. Quadratic Harnesses}

Let us recall, following [5] that $\mathrm{QH}$ is a stochastic process $\left\{X_{t}\right\}_{t \geq 0}$ defined for $t \geq 0$ on a certain probability space $(\Omega, \mathcal{F}, P)$ satisfying the following definition:

Definition 1. A stochastic process $\left\{X_{t}\right\}_{t \geq 0}$ will be called quadratic harness if the following 4 conditions are satisfied:

1. $X_{0}=0, \forall t \geq 0, E X_{t}=0$,

2. $\forall s, t \geq 0, E X_{s} X_{t}=\min (s, t)$,

3. $\forall 0 \leq s<t<u: E\left(X_{t} \mid \mathcal{F}_{s, u}\right)=\frac{u-t}{u-s} X_{s}+\frac{t-s}{u-s} X_{u}$, a.s.

4. $\forall 0 \leq s<t<u: E\left(X_{t}^{2} \mid \mathcal{F}_{s, u}\right)=Q_{s, t, u}\left(X_{s}, X_{u}\right)$,

where $Q_{s, t, u}(x, y)$ is a certain quadratic form determined by 6 coefficients and $\mathcal{F}_{s, u}=\sigma\left(X_{t}: t \in(0, s] \cup[u, \infty)\right)$.

Bryc, Matysiak, Wesołowski showed in [5] that there exist 5 parameters which they denoted by $\tau, \sigma, \theta, \eta, q$ such that the quadratic form $Q$ is completely determined i.e. respective coefficients are defined by the known functions of $s, t, u$ and $\tau, \sigma, \theta, \eta, q$. Bryc, Matysiak, Wesołowski deduced that $\sigma, \tau \geq 0, q \leq 1+2 \sqrt{\sigma \tau}$ and $\eta, \theta \in \mathbb{R}$. More precisely they showed that

$Q_{s, t, u}(x, y)=A(s, t, u) x^{2}+B(s, t, u) x y+C(s, t, u) y^{2}+D(s, t, u) x+E(s, t, u) y+F(s, t, u)$, where

$$
\begin{aligned}
A(s, t, u) & =\frac{(u-t)(u(1+\sigma t)+\tau-q t)}{(u-s)(u(1+\sigma s)+\tau-q s)}, \\
B(s, t, u) & =\frac{(u-t)(t-s)(1+q)}{(u-s)(u(1+\sigma s)+\tau-q s)}, \\
C(s, t, u) & =\frac{(t-s)(t(1+\sigma s)+\tau-q s)}{(u-s)(u(1+\sigma s)+\tau-q s)}, \\
D(s, t, u) & =\frac{(u-t)(t-s)(u \eta-\theta)}{(u-s)(u(1+\sigma s)+\tau-q s)}, \\
E(s, t, u) & =\frac{(u-t)(t-s)(-s \eta+\theta)}{(u-s)(u(1+\sigma s)+\tau-q s)}, \\
F(s, t, u) & =\frac{(u-t)(t-s)}{(u(1+\sigma s)+\tau-q s)} .
\end{aligned}
$$

The authors were seeking quadratic harnesses that are also Markov processes and assuming the existence of all moments they were trying to find a family of orthogonal polynomials $\left\{p_{n}(x ; t)\right\}_{t \geq 0, n \geq-1}$ such that

$$
\forall n \geq 0, t>s \geq 0: E\left(p_{n}\left(X_{t} ; t\right) \mid \mathcal{F}_{\leq s}\right)=p_{n}\left(X_{s} ; s\right) \text {, a.s. }
$$


Such family of QH that are also Markov will be called MQH and obviously they constitute a subset of all $\mathrm{QH}$.

Family of orthogonal polynomials of $\mathrm{MQH}$ will be called orthogonal martingale polynomials (briefly OM family of polynomials of the $\mathrm{MQH}\left\{X_{t}\right\}$ ).

Obviously we have $p_{-1}(x, t)=0, p_{0}(x ; t)=1$. Moreover the authors show in [5] that $p_{1}(x ; t)=x$. Now recall that following general theory of orthogonal polynomials presented e.g. in 9 or in 10 that every family of orthogonal polynomials $\left\{r_{n}(x)\right\}$ satisfies the so called 3 -term recurrence (1.7), i.e. the product $x r_{n}(x)$ is a linear combination of $r_{k}$ for $k=n+1, n, n-1$.

Note that if (1.7) and (A.7) are to make sense we must have $a_{n}(t)>0$ for all $t$ and $n>-1$. Moreover from the general theory of orthogonal polynomials it follows that if $a_{n-1}(t) c_{n}(t) \geq 0$ for all $n$ then the measure with respect to which polynomials $p_{n}$ are to be orthogonal is nonnegative i.e. polynomials have probabilistic interpretation. Hence it is reasonable to consider only such $\mathrm{QH}$ for which this condition is satisfied for all $n>-1$ and $t \geq 0$.

Bryc, Matysiak, Wesołowski showed also in the same paper that coefficients $a_{n}$, $b_{n}, c_{n}$ must be linear functions of $t$. This an easy conclusion of the condition 3 . of the Definition 1. For the sake of completeness we will prove this fact.

Proposition 4. Let $\left\{X_{t}\right\}_{t \geq 0}$ be $M Q H$ with parameters $\sigma, \tau, \theta, \eta, q$ such that $\forall t>0$ : supp $X_{t}$ contains infinite number of points. Let $\left\{p_{n}(x ; t)\right\}_{n \geq 0}$ denote its family of OM polynomials. Then

i) $\forall n>0 p_{n}(0,0)=0$ consequently $\forall n>0: E\left(p_{n}\left(X_{t} ; t\right)\right)=0$ thus polynomials $p_{n}$ constitute the family of orthogonal polynomials of the marginal distribution i.e. distribution of $X_{t}$.

ii) There must exist six number sequences $\left\{\alpha_{n}\right\},\left\{\beta_{n}\right\},\left\{\gamma_{n}\right\},\left\{\delta_{n}\right\},\left\{\varepsilon_{n}\right\},\left\{\varphi_{n}\right\}$ such that:

$$
a_{n}(t)=\alpha_{n} t+\beta_{n}, b_{n}(t)=\gamma_{n} t+\delta_{n}, c_{n}(t)=\varepsilon_{n} t+\varphi_{n},
$$

with $\alpha_{0}=0, \beta_{0}=1, \gamma_{0}=0, \delta_{0}=0, \varepsilon_{1}=1, \varphi_{1}=0$.

Proof of Proposition 4. i) First of all notice that from (A.7) it follows that $\forall n>-1$ $E p_{n}\left(X_{t}, t\right)=E p_{n}(0,0)=\xi_{n}$ a constant that does not depend on $t$. Secondly notice that $a_{n}(0)=\beta_{n}, b_{n}(0)=\delta_{n}, c_{n}(0)=\varphi_{n}$. Further notice that following (1.7) these constants satisfy the following second order recursion:

$$
\xi_{n+1}=-\frac{\delta_{n}}{\beta_{n}} \xi_{n}-\frac{\varphi_{n}}{\beta_{n}} \xi_{n-1},
$$

with $\xi_{-1}=0, \xi_{0}=1$. Besides we also have $0=\beta_{0} p_{1}(0 ; 0)+\delta_{0} p_{0}(0 ; 0)+\varphi_{0} p_{-1}(0 ; 0)$. Hence we deduce that $p_{1}(0,0)=0$, that is $\xi_{1}=0$. Now notice that if we chose $\varphi_{1}=0$ then we would have $\xi_{2}=0$ that is two successive constants $\xi_{n}$ being equal to zero consequently all must be equal to zero. Thus the choice $\varphi_{1}=0$ enables to select sequence $\left\{p_{n}\right\}$ to be both $\mathrm{OM}$ and have the property that $E p_{n}\left(X_{t}, t\right)=0$. On the other hand since $E X_{t}^{2}=t$ we take $n=1$ in (1.7) and use the fact that $E p_{n}\left(X_{t}, t\right)=0$ and deducing that $\varepsilon_{1}=1$. Sequence $\left\{p_{n}\right\}$ is thus a sequence of orthogonal polynomials that for some measure $\mu$ satisfy $\int p_{n} d \mu=0$ for all $n>0$. Since we have also 3 -term recurrence satisfied by polynomials $p_{n}$ we deduce that also $\int x p_{n} d \mu=0$ for all $n>1$. Similarly we deduce that $\int x^{k} p_{n} d \mu=0$ for all $n>k$. Hence polynomials must constitute family of orthogonal polynomials of measure $\mu$. 
ii) On one hand we have: $E\left(X_{t} p_{n}\left(X_{t} ; t\right) \mid \mathcal{B}_{\leq s}\right)=a_{n}(t) p_{n+1}\left(X_{s} ; s\right)+b_{n}(t) p_{n}\left(X_{s} ; s\right)$ $+c_{n}(t) p_{n-1}\left(X_{s} ; s\right)$. On the other:

$$
\begin{gathered}
E\left(X_{t} p_{n}\left(X_{t} ; t\right) \mid \mathcal{B}_{\leq s}\right)=E\left(X_{t} p_{n}\left(X_{u} ; u\right) \mid \mathcal{B}_{\leq s}\right)=E\left(E\left(X_{t} \mid \mathcal{B}_{\leq s, \geq u}\right) p_{n}\left(X_{u} ; u\right) \mid \mathcal{B}_{\leq s}\right)= \\
\frac{(u-t)}{u-s} X_{s} p_{n}\left(X_{s} ; s\right)+\frac{t-s}{u-s} E\left(X_{u} p_{n}\left(X_{u} ; u\right) \mid \mathcal{B}_{\leq s}\right) \\
=\frac{(u-t)}{u-s}\left(a_{n}(s) p_{n+1}\left(X_{s} ; s\right)+b_{n}(s) p_{n}\left(X_{s} ; s\right)+c_{n}(s) p_{n-1}\left(X_{s} ; s\right)\right) \\
+\frac{t-s}{u-s}\left(a_{n}(u) p_{n+1}\left(X_{s} ; s\right)+b_{n}(u) p_{n}\left(X_{s} ; s\right)+c_{n}(u) p_{n-1}\left(X_{s} ; s\right)\right) .
\end{gathered}
$$

Comparing appropriate coefficients we get:

$$
a_{n}(t)=\frac{(u-t)}{u-s} a_{n}(s)+\frac{t-s}{u-s} a_{n}(u)
$$

and similarly for $b_{n}(t)$ and $c_{n}(t)$. Now set $s=0$ in (A.8). Remembering that we had $a_{n}(0)=\beta_{0}$ and subtracting from both sides $\beta_{0}$ we get

$$
\frac{a_{n}(t)-\beta_{0}}{t}=\frac{a_{n}(u)-\beta_{0}}{u},
$$

from which we deduce that $a_{n}(t)$ must be linear function of $t$. We argue similarly for other coefficients $b_{n}(t)$ and $c_{n}(t)$.

Hence these coefficients are defined in fact by 6 families of sequences. More precisely we will seek relationships between families of numbers that are implied by the conditions that MQH's must satisfy.

We have the following theorem that is a version of the result of [5]. More precisely since our definitions of the numbers parameters $\left\{\alpha_{n}\right\},\left\{\beta_{n}\right\},\left\{\gamma_{n}\right\},\left\{\delta_{n}\right\},\left\{\varepsilon_{n}\right\}$, $\left\{\varphi_{n}\right\}$ is other than that the ones from [5] the resulting equations differ slightly but are basically the same. We believe that our notation is more logical but as usually it is in fact a matter of taste. Besides the proof that we are presenting is much simpler both conceptually and mathematically than the result of in [5. Nevertheless it is relatively long.

Theorem 3. Assuming that process $\left\{X_{t}\right\}_{t>0}$ is a MQH with parameters $\sigma, \tau, \theta, \eta, q$ and family of polynomials $\left\{p_{n}\right\}$ constitute its family of OM polynomials. Then families of numbers $\left\{\alpha_{n}\right\},\left\{\beta_{n}\right\},\left\{\gamma_{n}\right\},\left\{\delta_{n}\right\},\left\{\varepsilon_{n}\right\},\left\{\varphi_{n}\right\}$ satisfy the following system of 5 recurrences given by (1.1)-(1.6).

Proof of Theorem 3. First of all notice that starting from : $x p_{n}(x ; t)=\left(\alpha_{n} t+\right.$ $\left.\beta_{n}\right) p_{n+1}(x ; t)+\left(\gamma_{n} t+\delta_{n}\right) p_{n}(x ; t)+\left(\varepsilon_{n} t+\varphi_{n}\right) p_{n-1}(x ; t)$. Iterating it we get:

$$
\begin{gathered}
x^{2} p_{n}(x ; t)= \\
\left(\alpha_{n} t+\beta_{n}\right)\left(\alpha_{n+1} t+\beta_{n+1}\right) p_{n+2}(x ; t)+\left(\left(\alpha_{n} t+\beta_{n}\right)\left(\gamma_{n+1} t+\delta_{n+1}\right)\right. \\
\left.+\left(\gamma_{n} t+\delta_{n}\right)\left(\alpha_{n} t+\beta_{n}\right)\right) p_{n+1}(x ; t) \\
+\left(\left(\alpha_{n} t+\beta_{n}\right)\left(\varepsilon_{n+1} t+\varphi_{n+1}\right)+\left(\gamma_{n} t+\delta_{n}\right)\left(\gamma_{n} t+\delta_{n}\right)\right. \\
\left.+\left(\varepsilon_{n} t+\varphi_{n}\right)\left(\alpha_{n-1} t+\beta_{n-1}\right)\right) p_{n}(x ; t) \\
+\left(\left(\gamma_{n} t+\delta_{n}\right)\left(\varepsilon_{n} t+\varphi_{n}\right)+\left(\varepsilon_{n} t+\varphi_{n}\right)\left(\gamma_{n-1} t+\delta_{n-1}\right)\right) p_{n-1}(x ; t) \\
+\left(\varepsilon_{n} t+\varphi_{n}\right)\left(\varepsilon_{n-1} t+\varphi_{n-1}\right) p_{n-2}(x ; t) .
\end{gathered}
$$


On one hand we get:

$$
\begin{gathered}
E\left(X_{t}^{2} p_{n}\left(X_{t} ; t\right) \mid \mathcal{B}_{\leq s}\right)=\left(\alpha_{n} t+\beta_{n}\right)\left(\alpha_{n+1} t+\beta_{n+1}\right) p_{n+2}\left(X_{s} ; s\right) \\
\left(\left(\alpha_{n} t+\beta_{n}\right)\left(\gamma_{n+1} t+\delta_{n+1}\right)+\left(\gamma_{n} t+\delta_{n}\right)\left(\alpha_{n} t+\beta_{n}\right)\right) p_{n+1}\left(X_{s} ; s\right) \\
+\left(\left(\alpha_{n} t+\beta_{n}\right)\left(\varepsilon_{n+1} t+\varphi_{n+1}\right)+\left(\gamma_{n} t+\delta_{n}\right)\left(\gamma_{n} t+\delta_{n}\right)+\left(\varepsilon_{n} t+\varphi_{n}\right)\left(\alpha_{n-1} t+\beta_{n-1}\right)\right) p_{n}\left(X_{s} ; s\right) \\
+\left(\left(\gamma_{n} t+\delta_{n}\right)\left(\varepsilon_{n} t+\varphi_{n}\right)+\left(\varepsilon_{n} t+\varphi_{n}\right)\left(\gamma_{n-1} t+\delta_{n-1}\right)\right) p_{n-1}\left(X_{s} ; s\right) \\
+\left(\varepsilon_{n} t+\varphi_{n}\right)\left(\varepsilon_{n-1} t+\varphi_{n-1}\right) p_{n-2}\left(X_{s} ; s\right)
\end{gathered}
$$

while on the other we get:

$$
\begin{gathered}
E\left(X_{t}^{2} p_{n}\left(X_{t} ; t\right) \mid \mathcal{B}_{\leq s}\right)=E\left(X_{t}^{2} p_{n}\left(X_{u} ; u\right) \mid \mathcal{B}_{\leq s}\right)=E\left(E\left(X_{t}^{2} \mid \mathcal{B}_{\leq s, \geq u}\right) p_{n}\left(X_{u} ; u\right) \mid \mathcal{B}_{\leq s}\right) \\
=A(s, t, u) X_{s}^{2} p_{n}\left(X_{s} ; s\right)+B(s, t, u) X_{s}\left(\left(\alpha_{n} u+\beta_{n}\right) p_{n+1}\left(X_{s} ; s\right)\right. \\
\left.\quad+\left(\gamma_{n} u+\delta_{n}\right) p_{n}\left(X_{s} ; s\right)+\left(\varepsilon_{n} u+\varphi_{n}\right) p_{n-1}\left(X_{s} ; s\right)\right) \\
+C(s, t, u)\left(\left(\alpha_{n} u+\beta_{n}\right)\left(\alpha_{n+1} u+\beta_{n+1}\right) p_{n+2}\left(X_{s} ; s\right)\right. \\
+\left(\left(\alpha_{n} u+\beta_{n}\right)\left(\gamma_{n+1} u+\delta_{n+1}\right)+\left(\gamma_{n} u+\delta_{n}\right)\left(\alpha_{n} u+\beta_{n}\right)\right) p_{n+1}\left(X_{s} ; s\right) \\
+\left(\left(\alpha_{n} u+\beta_{n}\right)\left(\varepsilon_{n+1} u+\varphi_{n+1}\right)+\left(\gamma_{n} u+\delta_{n}\right)\left(\gamma_{n} u+\delta_{n}\right)\right. \\
\left.\quad+\left(\varepsilon_{n} u+\varphi_{n}\right)\left(\alpha_{n-1} u+\beta_{n-1}\right)\right) p_{n}\left(X_{s} ; s\right) \\
+\left(\left(\gamma_{n} u+\delta_{n}\right)\left(\varepsilon_{n} u+\varphi_{n}\right)+\left(\varepsilon_{n} u+\varphi_{n}\right)\left(\gamma_{n-1} u+\delta_{n-1}\right)\right) p_{n-1}\left(X_{s} ; s\right) \\
\left.\quad+\left(\varepsilon_{n} u+\varphi_{n}\right)\left(\varepsilon_{n-1} u+\varphi_{n-1}\right) p_{n-2}\left(X_{s} ; s\right)\right) \\
+D(s, t, u) X_{s} p_{n}\left(X_{s} ; s\right)+E(s, t, u)\left(\left(\alpha_{n} u+\beta_{n}\right) p_{n+1}\left(X_{s} ; s\right)\right. \\
+\left(\gamma_{n} u+\delta_{n}\right) p_{n}\left(X_{s} ; s\right)+\left(\varepsilon_{n} u+\varphi_{n}\right) p_{n-1}\left(X_{s} ; s\right)+F(s, t, u) p_{n}\left(X_{s} ; s\right)
\end{gathered}
$$

Comparing coefficients by respectively $p_{n+i}\left(X_{s} ; s\right), i=2,-2,1,-1,0$ we get:

$0=\left(\alpha_{n} t+\beta_{n}\right)\left(\alpha_{n+1} t+\beta_{n+1}\right)-A(s, t, u)\left(\alpha_{n} s+\beta_{n}\right)\left(\alpha_{n+1} s+\beta_{n+1}\right)$

$$
-B(s, t, u)\left(\alpha_{n} u+\beta_{n}\right)\left(\alpha_{n+1} s+\beta_{n}\right)-C(s, t, u)\left(\alpha_{n} u+\beta_{n}\right)\left(\alpha_{n+1} u+\beta_{n+1}\right)
$$

from which it follows that (by Mathematica):

$$
\tau \alpha_{n} \alpha_{n+1}-\alpha_{n+1} \beta_{n}+q \alpha_{n} \beta_{n+1}+\sigma \beta_{n} \beta_{n+1}=0 .
$$

Similarly we get the remaining equations.

Department of Mathematics and Information Sciences,, Warsaw University of TechNOLOGy, ul Koszykowa 75, 00-662 Warsaw, Poland

E-mail address: pawel.szablowski@gmail.com 